Original scientific paper

\title{
MECHANISM OF ROAD CAPACITY UNDER DIFFERENT PENETRATION SCENARIOS OF AUTONOMOUS VEHICLES
}

\author{
Zhang, L. Y.; Duan, X. K.; Ma, J.\#; Zhang, M.; Wen, Y. \& Wang, Y. \\ School of Civil Engineering, Suzhou University of Science and Technology, Suzhou 215011, China \\ E-Mail: 9764634@qq.com $\left({ }^{\#}\right.$ Corresponding author $)$
}

\begin{abstract}
This paper takes L0 (ordinary vehicles), L2 and L4 (autonomous vehicles) as the research objects, adopts different car-following rules and improves them respectively based on driver's personality factors and variable time headway (VTH) strategy, introduces a benefit parameters to distinguish lane changing ability of them, and evaluates road capacity under mixed traffic flow with a basic diagram model and average travel time. Use SUMO to build a simulation platform and conduct a real-time systematic research based on Python. The results prove that: (1) After the penetration rate of autonomous exceeds $60 \%$, road capacity can be effectively improved, and the maximum increase of $32.52 \%$ occurs in $100 \%$ penetration scenario. (2) When traffic density is less than 27 vehicles $/ \mathrm{km}$, the average speed continues to be the maximum in $100 \%$ scenario, and when it is greater than 27 vehicles $/ \mathrm{km}$, the critical penetration scenario is $80 \%$. (3) The average travel time begins to decrease after the penetration rate exceeds $20 \%$, and can be reduced by $23.38 \%$ in $100 \%$ scenario. It shows that traffic efficiency is closely associated with penetration rates of autonomous vehicles.

(Received in October 2021, accepted in February 2022. This paper was with the authors 1 month for 1 revision.)
\end{abstract}

Key Words: Autonomous Vehicles; Road Capacity, Mixed Traffic Flow, SUMO, Penetration Rate

\section{INTRODUCTION}

As the fusion of vehicles and technologies like artificial intelligence, autonomous will become an inevitable trend of modern traffic development. However, the full popularization of automation will not be achieved overnight, the traffic flow is bound to experience a gradual increase of autonomous. Scale of the quantity of autonomous on the road to the total vehicles is known as autonomous vehicles penetration. Mastering the impact of different penetration scenarios of autonomous on road capacity is the main problem we are facing, which will benefit the deployment and control of autonomous in the future.

Traffic simulation can reflect the real traffic phenomenon by testing various traffic scenarios, which is the most appropriate method to analyse the characteristics of vehicle driving behaviour [1,2]. Traffic simulation technology can be classified as macro, meso and micro according to the granularity [3,4]. In recent years, many scholars all over the world have studied the mixed traffic flow of autonomous penetration scenarios by building traffic simulation platforms and using microscopic models. For example, $\mathrm{Hu}$ et al. [5] conducted a sensitivity analysis on traffic benefit of hybrid traffic-flow infiltrating different scales of intelligent vehicles under peak and flat peak environments based on Vissim simulation. Lu et al. [6] considered the influence of autonomous penetration and level on traffic flow, and analysed the evolution law of macroscopic fundamental diagram (MFD) based on SUMO. Ye et al. [7] analysed the dynamic evolution law of traffic flow under heterogeneous flow scenarios with different penetration rates of autonomous. Kerschbaumer et al. [8], Yao et al. [9], and Arvin et al. [10] described driving behaviours of vehicles with different categories and levels through different models, and based on Vissim, analysed the interaction process of vehicles with different permeability under various road sections and special conditions.

It is not difficult to find that the penetration rate and level of autonomous are closely bound up with the evolution of traffic. Up to now, researches in this field have the following problems. First, the level of automation is generally limited to L1 L2, second, there are few investigations 
on hybrid multi-level autonomous. In view of the shortcomings of existing studies, this paper will be based on SUMO (Simulation of Urban Mobility) and combined with Python language to study the traffic capacity of different levels of autonomous in different penetration scenarios. However, since a single driving model cannot accurately account complex driver behaviours under actual conditions, this paper selects diverse following models respectively to its description, namely, the intelligent driver model (IDM) is applied to trace ordinary vehicles, and the Adaptive Cruise Control (ACC) model is used for depicting autonomous vehicles, and improving them with driver personality factors and variable time headway (VTH) strategies respectively. Meanwhile, based on LC2013 lane-changing model, the benefit parameters of autonomous driving level are introduced to distinguish the lane-changing ability by parameters values. Simulation results show that considering the influence of the driver's personality can better reflect the actual uncertain driving scenarios, the autonomous after integrating the VTH strategy can run more stably and the simulation is more appropriate. The improvement of the model can provide a real and reliable basis for the research on the road capacity mechanism of ordinary-multi-level autonomous mixed traffic flow in this paper.

\section{IMPACT OF DIFFERENT PENETRATION RATES OF AUTONOMOUS VEHICLES}

From the perspective of autonomous driving level and its technical development, autonomous vehicles can be regarded as a kind of machine that can control vehicles partially or completely without human operation by virtue of advanced vehicle sensors, controllers and actuators, and by integrating artificial intelligence and other technologies [11]. Autonomous is the vehicle's understanding, learning and materialization of the driver's "perception-decision-control" driving process. At present, there are four authoritative grading standards for autonomous technology in the world, including NHTSA, SAE, ERTRAC, and SAE-China [12]. Among them, the SAE standard is the most detailed and specific, as shown in Table I.

Table I: SAE classification standard.

\begin{tabular}{|c|c|c|c|c|c|c|}
\hline $\begin{array}{c}\text { Automation } \\
\text { level }\end{array}$ & Name & Class definition & $\begin{array}{l}\text { Driving } \\
\text { control }\end{array}$ & $\begin{array}{c}\text { Environmental } \\
\text { monitoring }\end{array}$ & $\begin{array}{c}\text { Take } \\
\text { over } \\
\text { object }\end{array}$ & $\begin{array}{l}\text { System } \\
\text { scope }\end{array}$ \\
\hline L0 & $\begin{array}{l}\text { Manual } \\
\text { driving }\end{array}$ & $\begin{array}{l}\text { The whole process is } \\
\text { controlled by driver, the } \\
\text { system only provides } \\
\text { auxiliary information }\end{array}$ & driver & \multirow{3}{*}{ driver } & \multirow{4}{*}{ driver } & none \\
\hline L1 & $\begin{array}{c}\text { Driving } \\
\text { assistance }\end{array}$ & $\begin{array}{l}\text { Perform one operation of } \\
\text { steering or acceleration and } \\
\text { deceleration, others are } \\
\text { controlled by driver }\end{array}$ & $\begin{array}{l}\text { driver } \\
\text { and } \\
\text { system }\end{array}$ & & & \multirow{4}{*}{ part } \\
\hline L2 & $\begin{array}{c}\text { Partially } \\
\text { automation }\end{array}$ & $\begin{array}{l}\text { Perform multiple operations } \\
\text { in steering or acceleration } \\
\text { and deceleration, others are } \\
\text { controlled by driver }\end{array}$ & \multirow{4}{*}{ system } & & & \\
\hline L3 & $\begin{array}{l}\text { Conditional } \\
\text { automation }\end{array}$ & $\begin{array}{l}\text { The system does most of the } \\
\text { operations, but driver needs } \\
\text { to be ready to respond to } \\
\text { system requests }\end{array}$ & & \multirow{3}{*}{ system } & & \\
\hline L4 & $\begin{array}{c}\text { Highly } \\
\text { automation }\end{array}$ & $\begin{array}{l}\text { The system does all driving } \\
\text { has environmental } \\
\text { limitations }\end{array}$ & & & \multirow{2}{*}{ system } & \\
\hline L5 & $\begin{array}{c}\text { Fully } \\
\text { automation }\end{array}$ & $\begin{array}{l}\text { The system completes all } \\
\text { driving operations without } \\
\text { driver intervention }\end{array}$ & & & & all \\
\hline
\end{tabular}


L0 (ordinary vehicle), which means that the vehicle is only equipped with a alerting system to warn drivers. L1 L2 belong to auxiliary driving, the automatic driving system only plays an auxiliary role, ADAS represented by L1 and L2 in the market is technically mature now, but the L1 can only control the vehicle in one direction, while the L 2 can control both horizontally and vertically. L3 belongs to conditional automatic driving, which requires the driver to respond to the system's takeover request in a timely manner at critical moments, involving information exchange and control authority switching between driver and system. L4 is highly autonomous driving, the driver does not need to observe the surrounding environment, the difference from the L5 is that the L4 is limited by the design operating conditions ODD, while the L5 does not.

Therefore, in terms of the driving subject alone, the driving subject of L0 L3 is driver, and L4 L5 is system. The driver can only adjust himself based on the subjective judgment, this uncertainty makes them behave differently in the process of controlling the vehicle. Since the automatic driving system can reduce the randomness, uncertainty and variability, the overall response level to the surrounding environment can be improved.

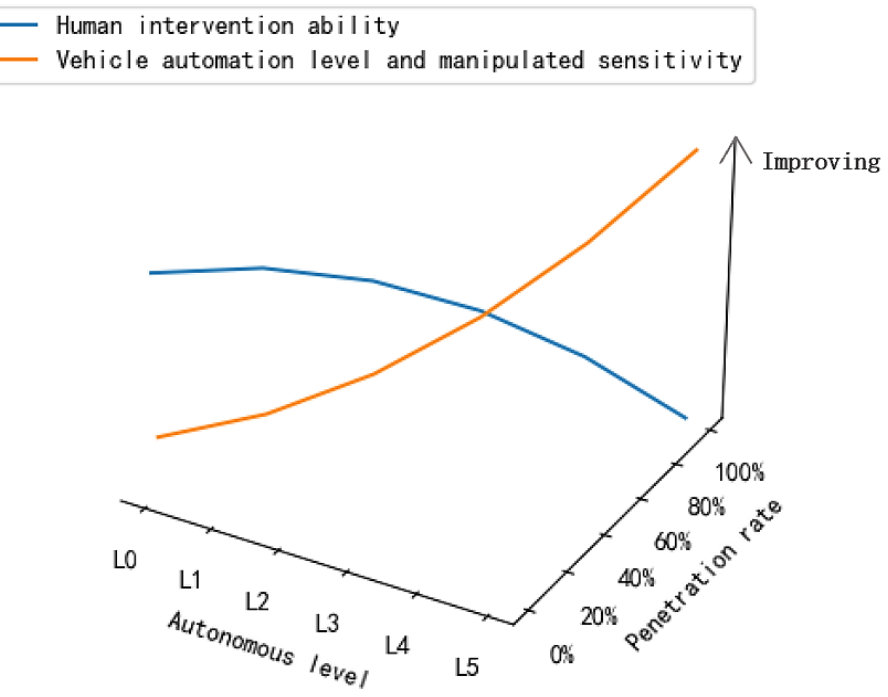

Figure 1: The impact of autonomous level and penetration rate on driving subject.

Fig. 1 shows the abstract impact of changes in automatic driving level and penetration rate on driving subjects, which shows that with the improvement of vehicle automatic driving levels and the increase of penetration rates, on the one hand, human intervention ability gradually decrease, on the other hand, due to the increasing dependence of vehicles on advanced driver assistance systems, there are more and more requirements on sensor combination, control effect of automatic driving system, vehicle communication and other functions [13], and the overall automation level of the road and system control sensitivity has been improved.

However, it still takes a long time for L5 production applications in the market. Therefore, this paper will take L2 and L4 as the research objects, and use the influence of different grades of autonomous on vehicles as the main basis to distinguish the different levels of vehicles driving characteristics.

\section{RESEARCH MODEL}

Vehicle following and lane changing are the process of vehicle speed adjustment and lane selection, which are the core driving behaviours in microscopic traffic flow [14]. The platform has implemented 15 car-following models, such as Krauss, ACC/CACC, IDM, and 3 lanechanging models, such as LC2013 [15]. These models analyse the interactions between vehicles from different perspectives based on different principles and through different parameters. 


\subsection{Car-following models under different penetration rates of autonomous vehicles}

(1) Ordinary car-following model

Intelligent driver model (IDM) [16] was originally presented by Treiber through a large number of experiments, since it was proposed, it has been widely used in the research on the car-following behaviour [17]. The model used speed and distance between itself and the preceding vehicle as bases for decision-making, and output its own acceleration [18]. Described as follows:

$$
\begin{gathered}
a_{n}(t)=a_{0}\left(1-\left(\frac{v_{n}(t)}{v_{0}}\right)^{\delta}-\left(\frac{s^{*}\left(v_{n}(t), \Delta v_{n}(t)\right)}{s_{n}(t)}\right)^{2}\right) \\
s_{n}(t)=x_{n-1}(t)-x_{n}(t)-l \\
\Delta v_{n}(t)=v_{n}(t)-v_{n-1}(t) \\
s^{*}\left(v_{n}(t), \Delta v_{n}(t)\right)=s_{0}+\max \left(0, v_{n}(t) T+\frac{v_{n}(t) \Delta v_{n}(t)}{2 \sqrt{a_{0} b}}\right)
\end{gathered}
$$

Here, $a_{n}(t)$ : acceleration of the following car; $a_{0}$ : maximum acceleration of the following car; $v_{n}(t)$ : speed of the following car; $v_{0}$ : desired speed; $\delta$ : acceleration index; $s^{*}\left(v_{n}(t), \Delta v_{n}(t)\right)$ : expected space headway of the following car; $s_{n}(t)$ : actual space headway between itself and the preceding car; $x_{n}(t)$ : following car displacement; $x_{n-1}(t)$ : preceding car displacement; $l$ : length of the car; $\Delta v_{n}(t)$ : speed difference between itself and the preceding car; $v_{n-1}(t)$ : speed of the preceding car; $s_{0}$ : minimum space headway in congested state; $T$ : minimum safe time headway; $b$ : comfortable deceleration.

Some studies have shown that the driver behaviour is the primary influencing factor of traffic accidents [19]. Drivers with different personalities will have different reactions and decisions when facing same traffic scenes, which directly affects driving conditions. Therefore, the driver's personality factors should be fully considered when modelling. Generally speaking, the driver's personality can be divided into aggressive, steady and cautious, in order to distinguish the difference of them, this paper introduces the parameter $\lambda$ into the calculation of the speed difference between two adjacent cars to represent the driver's risk degree. Typically, the driver adjusts its speed based on the relative conditions (speed and distance) between itself and the preceding vehicle [4], $\lambda_{i} v_{i}$ represents his estimated speed of the preceding vehicle, a higher $\lambda$ means a more adventurous driver. According to the relevant literature [20], $\lambda \in$ $[0.7,0.9]$. Described as follows:

$$
\Delta v_{n}(t)=v_{n}(t)-\lambda v_{n-1}(t) \quad \lambda= \begin{cases}0.7 & \text { (cautious) } \\ 0.8 & \text { (steady) } \\ 0.9 & \text { (aggressive) }\end{cases}
$$

The model comparison results after considering driver's personalities are proved in Fig. 2.

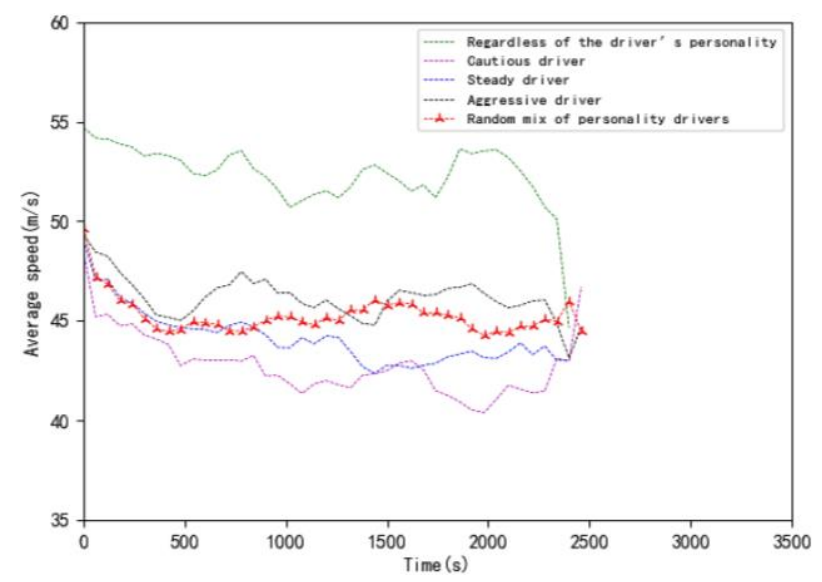

Figure 2: Comparison of car-following models of ordinary vehicles. 
Fig. 2 shows that the average speed of vehicles after considering influence of driver's personalities is reduced, indicating that driver's personalities can indeed affect the running state of vehicles. Among them, the average speed of all cautious drivers is lower than that of aggressive drivers, and drivers who randomly mix various personalities are located between the two situations, which is more tally with the actual situation.

(2) Autonomous car-following model

Autonomous driving is to replace the driver to control the vehicle through the automatic driving technology, and the realization of the control requires the cooperation of multiple technologies. Among them, the speed and distance control of vehicles are the key technologies to realize the longitudinal control. Based on the related researches of Milanés, Shladover, Xiao et al. [21-23], this paper proposes an ACC car-following model considering the variable time headway (VTH) strategy. According to Xiao's [23] research results, the control strategies of the model are fallen into four types: speed, gap, gap-closing and collision avoidance.

When sensors senses that there is no car ahead or the distance between the two adjacent cars is greater than $120 \mathrm{~m}$, it will take the preset speed as the control target, drive the preset and realtime speed error as the control variable. Described as follows:

$$
a_{n}(t+1)=k_{1}\left(v_{d}-v_{n}(t)\right)
$$

Here, $a_{n}(t+1)$ : acceleration of the following car at the next time; $v_{d}$ : desired speed; $v_{n}(t)$ : speed of the following car; $k_{1}$ : control gain of determining the speed deviation rate for acceleration, $k_{1}=0.4 \mathrm{~s}^{-1}[23]$.

When distance and speed deviation are short of $0.2 \mathrm{~m}$ and $0.1 \mathrm{~m} / \mathrm{s}$ at the same time, the vehicle's acceleration at the next moment will be adjusted according to the distance and speed deviation from the preceding vehicle. Described as follows:

$$
\begin{gathered}
a_{n}(t+1)=k_{2} e_{n}(t)+k_{3}\left(v_{n-1}(t)-v_{n}(t)\right) \\
e_{n}(t)=x_{n-1}(t)-x_{n}(t)-d_{0}-t_{d} v_{n}(t)
\end{gathered}
$$

Here, $e_{n}(t)$ : distance error between the following and preceding car; $x_{n-1}(t)$ : displacement of the preceding car; $x_{n}(t)$ : displacement of the following car; $d_{0}$ : minimum safe distance; $t_{d}$ : preset constant time headway; $v_{n-1}(t)$ : speed of the preceding car; $v_{n}(t)$ : speed of the following car; $k_{2}, k_{3}$ : control gains for position and velocity deviations, $k_{3}=0.23 \mathrm{~s}^{-2}, k_{3}=0.07 \mathrm{~s}^{-1}$ [23].

When space between two adjacent vehicles is short of $100 \mathrm{~m}$, by modifying the parameters in the gap control strategy, that is, let $k_{2}$ and $k_{3}$ be $0.04 \mathrm{~s}^{-2}$ and $0.8 \mathrm{~s}^{-1}$ respectively to achieve the gap-closing control strategy. When space is among $100 \mathrm{~m}$ and $120 \mathrm{~m}$, it is converted to the previous control strategy. When the distance is under $100 \mathrm{~m}$, distance deviation is negative, and speed deviation is inferior to $0.1 \mathrm{~m}^{-1}$, let $k_{2}$ and $k_{3}$ be $0.8 \mathrm{~s}^{-2}$ and $0.23 \mathrm{~s}^{-1}$ respectively to realize the collision avoidance control strategy.

The determination of the safety distance is the most important link in ACC model. The existing spacing control incorporates constant and variable. The variable strategy is further segmented into constant time headway $(\mathrm{CTH})$ and variable time headway (VTH).

The spacing control in the original ACC model adopts the variable spacing strategy of CTH. However, in real life, the CTH strategy cannot cope with some complicated situations. Therefore, this paper adopts the VTH strategy based on the original ACC model. The VTH strategy means that the time headway will vary with the speed and acceleration of the front vehicle. Yanakiev and Kanellakopoulos [24] considered the influence of relative speed of the two vehicles on headway, and uses the saturation function to make the headway stay within a reasonable range. Described as follows:

$$
t_{h}=\operatorname{sat}\left(t_{0}-k_{v} v_{r}\right)=\left\{\begin{array}{cc}
t_{h, \max } & t_{0}-k_{v} v_{r}>t_{h, \max } \\
t_{0}-k_{v} v_{r}, & 0<t_{0}-k_{v} v_{r}<1 \\
0 & \text { otherwise }
\end{array}\right.
$$


Here, $t_{h}$ : time headway; $t_{0}$ : basic time headway; $k_{v}$ : time headway parameter and greater than $0 ; v_{r}$ : the relative speed of two adjacent cars; sat(): saturation function, upper and lower limits $t_{h, \max }$ and 0 of the time headway are set by it. Described as follows:

$$
e_{n}(t)=x_{n-1}(t)-x_{n}(t)-d_{0}-t_{h} v_{n}(t)
$$

In this paper, based on the standard of space headway in flow, the model is compared and verified from the disturbance stability of car-following behaviour. Verify the simulation condition settings: start to set the original speed of the preceding vehicle at $30 \mathrm{~m} / \mathrm{s}$ at $100 \mathrm{~s}$ of simulation, take $20 \mathrm{~s}$ as the span, and the acceleration and deceleration are both $1 \mathrm{~m} / \mathrm{s}^{2}$, the vehicle ahead from the original velocity of $30 \mathrm{~m} / \mathrm{s}$ to slow down to $10 \mathrm{~m} / \mathrm{s}$, from $10 \mathrm{~m} / \mathrm{s}$ up to $30 \mathrm{~m} / \mathrm{s}$, the whole disturbance process is $350 \mathrm{~s}$ in total. Comparison results are shown in Fig. 3.

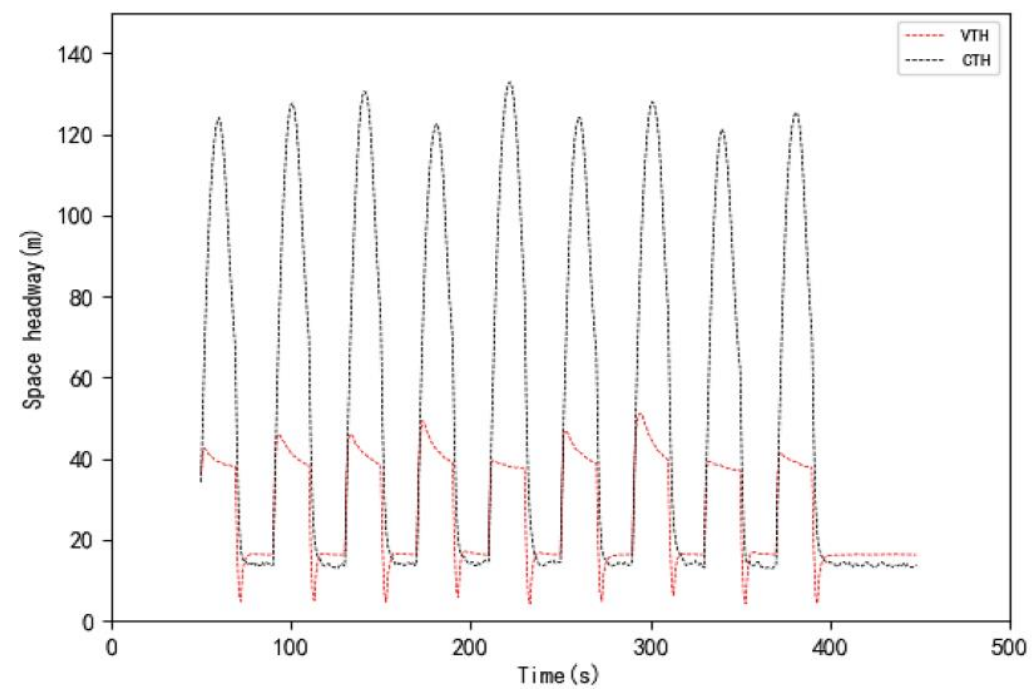

Figure 3: Comparison of car-following models of autonomous vehicles.

As can be seen from Fig. 3, contrasted to the original model, the current model using the VTH strategy has a smaller variation in space headway, indicating that the VTH strategy makes the vehicle more stable.

\subsection{Lane-changing model under different penetration rates of autonomous vehicles}

SUMO's default lane change model LC2013 obtains an effective path by calculating the occupancy rate of all lanes, the distance that can continue to travel without changing lanes, and the deviation value from the optimal lane, so as to perform lane change selection. The calculation method of the minimum lane change distance is described as follows [15]:

$$
d_{l c}(t)=\left\{\begin{array}{l}
v(t) \cdot \alpha_{1}+2 L \\
v(t) \cdot \alpha_{2}+2 L
\end{array}\right.
$$

Here, $d_{l c}(t)$ : the change lanes for the shortest distance; $v(t)$ : speed of the car; $\alpha_{1}, \alpha_{2}$ : the scale factor; $L$ : length of the car.

This model divides lane-changing behaviours into four types according to lane-changing purposes: strategic, cooperative, tactical and regulatory. Strategic change is an act performed under the condition that the present lane of the vehicle is not connected to the destination, cooperative change is an act performed to facilitate the normal driving of adjacent vehicles, tactical change is an act performed when one wants to pursue a high speed but is blocked by the slow speed of the ahead, regulatory change is an act performed to comply with traffic regulations. The parameter settings of different change behaviours have different effects on vehicles' lane-changing behaviour. Therefore, this paper introduces an abstract effect parameter 
$E T_{\theta}(g)$ (Effect Type (Gread)) based on parameters determined by lane-changing type to represent the influence of vehicles with different autonomous levels on lane-changing behaviours, where g refers to L0, L2 and L4. Described as follows:

$$
\begin{gathered}
E T_{\theta}(g) d_{l c}(t)=\left\{\begin{array}{l}
v(t) \cdot \alpha_{1}+2 L \\
v(t) \cdot \alpha_{2}+2 L
\end{array}\right. \\
E T_{\theta}(g)=E T_{\text {lcStrategic }}(g)+E T_{\text {lcSpeedGain }}(g)+E T_{\text {lcAssertive }}(g)+E T_{\text {lcCooPerative }}(g)
\end{gathered}
$$

In summary, the relevant parameters that affect autonomous levels are shown in Table II.

Table II: Related influencing parameters of driving behaviour model.

\begin{tabular}{|c|c|c|c|c|c|}
\hline \multirow{2}{*}{ Model } & \multirow{2}{*}{ Parameters } & Meaning & \multicolumn{3}{|c|}{ Value } \\
\cline { 4 - 6 } & minGap & Minimum distance between cars $(\mathrm{m})$ & 2.5 & 2 & 1.2 \\
\hline \multirow{4}{*}{$\begin{array}{c}\text { IDM/ } \\
\text { ACC }\end{array}$} & accel & Acceleration $\left(\mathrm{m} / \mathrm{s}^{2}\right)$ & 2.6 & 3.5 & 3.7 \\
\cline { 2 - 6 } & decel & Deceleration $\left(\mathrm{m} / \mathrm{s}^{2}\right)$ & 4.5 & 4.5 & 4.5 \\
\cline { 2 - 6 } & Sigma & Driving proficiency & 0.5 & 0.25 & 0 \\
\cline { 2 - 6 } & tau & Desired minimum time headway (Reaction time, s) & 1 & 0.9 & 0.6 \\
\hline \multirow{4}{*}{ LC2013 } & lcStrategic & $\begin{array}{c}\text { Strategic lane change willingness, the larger the value, the } \\
\text { earlier the change }\end{array}$ & 1 & 1.2 & 3 \\
\cline { 2 - 6 } & lcSpeedGain & $\begin{array}{c}\text { Tactical lane change willingness, the larger the value, the } \\
\text { more frequent changes }\end{array}$ & 1 & 1.2 & 5 \\
\cline { 2 - 6 } & lcAssertive & $\begin{array}{c}\text { Willingness to accept smaller vehicle clearances in the } \\
\text { target lane }\end{array}$ & 1 & 1.2 & 3.4 \\
\cline { 2 - 6 } & lcCooPerative & $\begin{array}{c}\text { The willingness to change lanes for cooperation, the } \\
\text { larger the value, the stronger the willingness to cooperate }\end{array}$ & 0.5 & 0.5 & 1 \\
\hline
\end{tabular}

For car-following behaviour, as the automatic level increases, the driver's imperfect parameters, minimum space headway and time headway will be significantly reduced. Starting from the L4, since the driving subject of vehicles are transformed from human to system, this paper sets L4 as defect-free, the intermediate value between it and L0 is taken as the L2. For lane-changing behaviour, Highly autonomous vehicles will show an increase in strategic forward-looking, tend to change lanes frequently in pursuit of high speed, the minimum safe distance required for lane-changing is smaller, the satisfaction value of staying in the right lane is low. Therefore, based on the performance of drivers and vehicles, and related studies, such as Kudarauskas [25], Erdmann [26], Lackey [27], Lu et al. [6] and Guériau and Dusparic [14], relevant parameter values are determined so that the established model can more accurately reflect the level of automatic driving.

\section{SIMULATION AND RESULTS}

\subsection{Simulation platform construction}

Based on SUMO, this paper builds a co-simulation platform with the help of the Python programming language to analyse the change rule of the road capacity of ordinary-multi-level autonomous mixed traffic flow. The interface principle is shown in Fig. 4. The platform construction method is simple and easy to implement, which is of great significance to the research related to traffic simulation.

There are three road modelling methods: one is to integrate the node file (node.xml), edge file (edge.xml), and additional file (add.xml) through netconvert command, the second is to import from the outside, such as OSM, Vissim, etc, the third is to draw directly through the 
netedit visual interface. Based on the netedit visualization interface, a $10 \mathrm{~km}$ one-way two-lane section is drawn in this paper, as shown in Fig. 5. Section 1 and Section 2 are both $1 \mathrm{~km}$ long, and Section 3 is $8 \mathrm{~km}$ long, which are used to represent common situations on expressways. Information collection is performed by adding E1 and E2 fixed detectors, and relevant speed limit information signs are set to control vehicle speed. Variable speed limit control is an effective management strategy to improve road safety [28], this paper adopts the previous method to make drivers choose driving speed within the specified range, for example, the design speeds of the lanes (running direction from left to right) are: $80 \mathrm{~km} / \mathrm{h}$ and $60 \mathrm{~km} / \mathrm{h}$.

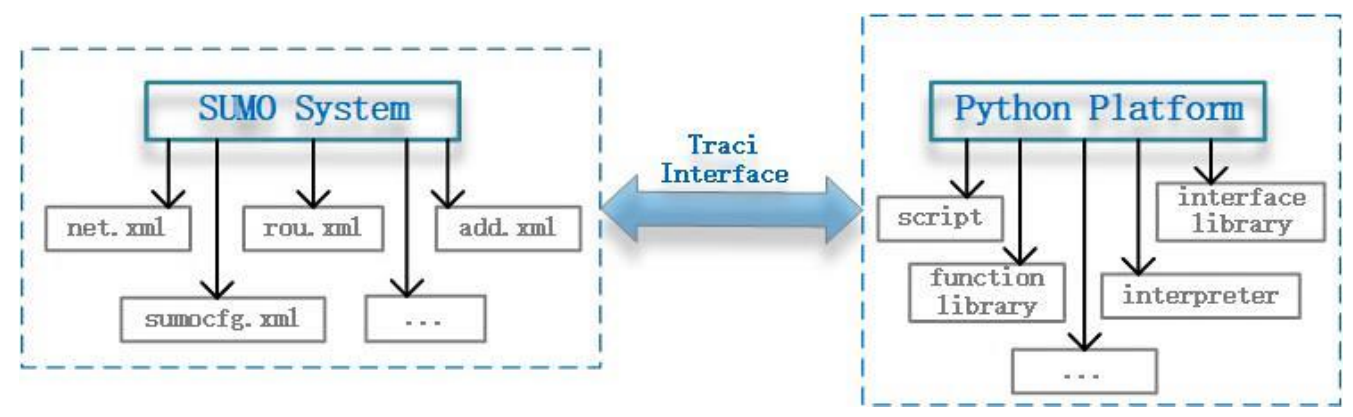

Figure 4: Principle of interface between SUMO and Python.

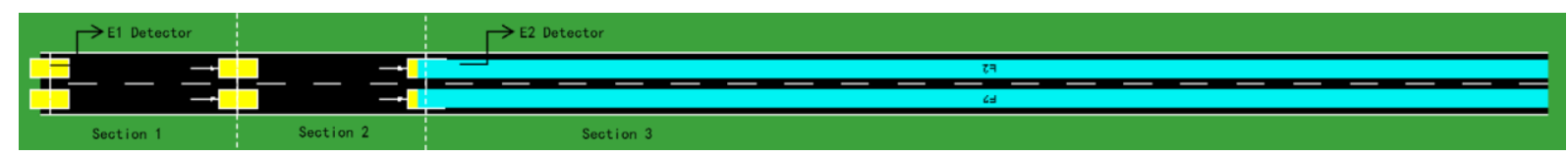

Figure 5: Road network model.

The construction of traffic simulation scenarios starts from two aspects: penetration rates and autonomous levels, as shown in Table III.

Table III: Simulation scene.

\begin{tabular}{|c|c|c|c|}
\hline \multirow{2}{*}{$\begin{array}{c}\text { Autonomous } \\
\text { penetration (P) }\end{array}$} & \multirow{3}{|c|}{ Vehicle composition } \\
\cline { 2 - 4 } & \multirow{2}{*}{ L0 (Ordinary vehicle) } & The composition of autonomous \\
\cline { 2 - 4 } & $100 \%$ & $\mathrm{~L} 2$ & $\mathrm{~L} 4$ \\
\hline $0 \%$ & $80 \%$ & $0 \%$ & $0 \%$ \\
\hline $20 \%$ & $60 \%$ & $10 \%$ & $10 \%$ \\
\hline $40 \%$ & $40 \%$ & $20 \%$ & $20 \%$ \\
\hline $60 \%$ & $20 \%$ & $30 \%$ & $30 \%$ \\
\hline $80 \%$ & $0 \%$ & $40 \%$ & $40 \%$ \\
\hline $100 \%$ & \multicolumn{3}{|c}{} \\
\hline
\end{tabular}

\subsection{Analysis of simulation results}

(1) Fundamental diagram analysis

The fundamental diagram is an accepted theory for macroscopically describing the dynamics of road traffic [29]. The density-flow, density-speed and maximum capacity improvement diagram under different autonomous permeability scenarios in the mixed flow of ordinary-multi-level autonomous are said in Figs. 6 and 7 separately. Where, the $P$ value represents permeabilities of all autonomous. 


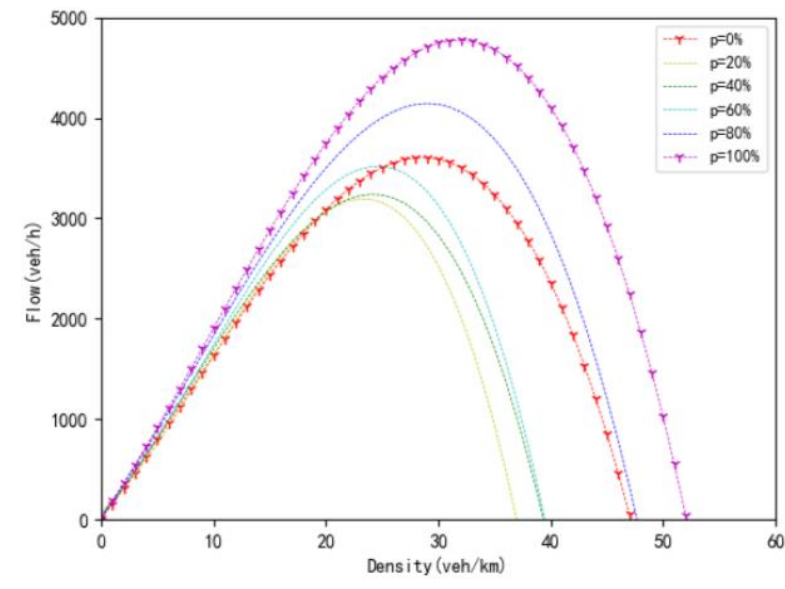

a) Density-flow diagram

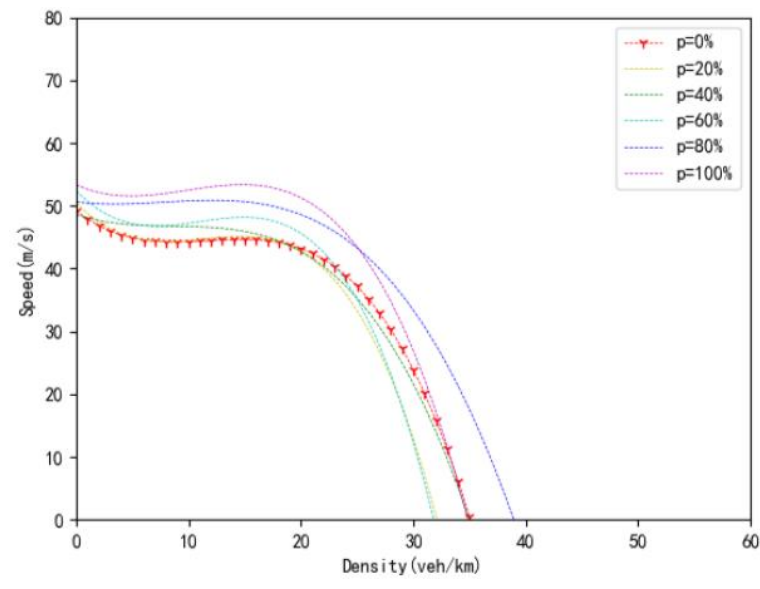

b) Density-speed diagram

Figure 6: Basic diagram under different penetration scenarios.

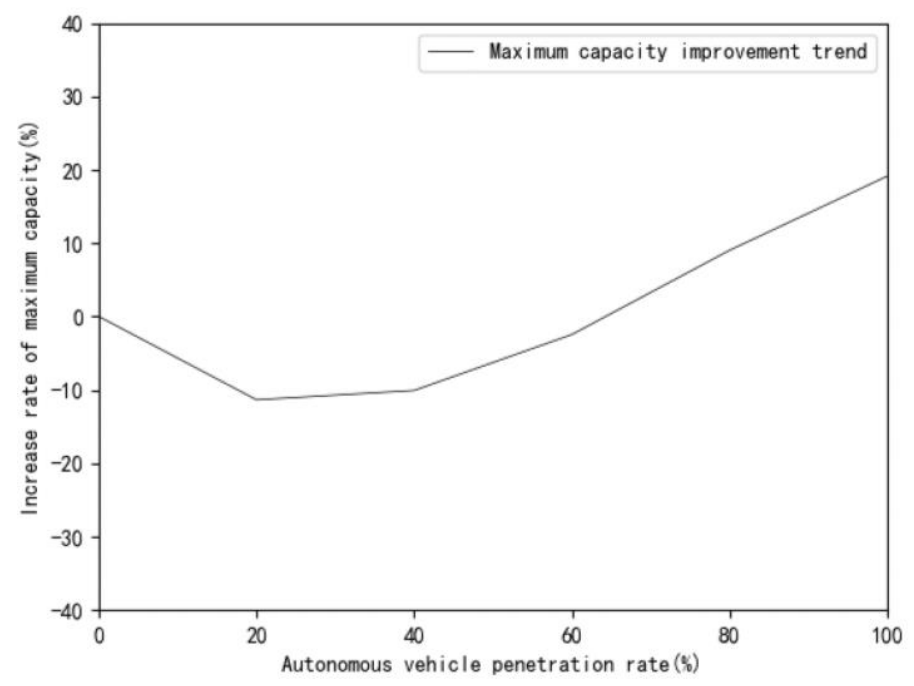

Figure 7: Maximum capacity under different penetration scenarios.

As demonstrated in Figs. 6 and 7, the density-flow and density-speed diagrams of autonomous in different proportions have almost the same variation tendency. Along with density increase, the average speed gradually decreases, and the flow first rises to the critical value and then gradually declines.

Under the same density, as the permeability of autonomous increases, the road capacity shows a tendency to climb up and then decline. When $P$ value is in a small range $(0 \% \sim 60 \%)$, the road capacity is reduced, but when $P$ value is greater than $60 \%$, the road capacity is significantly improved, especially when $P$ value is $100 \%$, That is, when all the vehicles on the road are autonomous, the capacity improvement reaches $32.52 \%$, which is the maximum value. However, for the average speed, under the same density, as long as there are autonomous, the overall average speed of the road will be improved, among them, when the density is less than 27 vehicles $/ \mathrm{km}$, it keeps the maximum value under the scenario of $100 \%$ autonomous, but when the density is greater than 27 vehicles $/ \mathrm{km}$, the critical penetration rate is $80 \%$. It is not difficult to see that the automatic driving technology can effectively improve the traffic capacity and efficiency, but the improvement strongly associated with autonomous driving permeability.

(2) average travel time

Table IV and Fig. 8 show the change trends of the average travel time and reduction value of vehicles beneath different permeability of autonomous. 
Table IV: Average vehicle travel time under different penetration scenarios of autonomous vehicles.

\begin{tabular}{|c|c|}
\hline Autonomous driving penetration (P) & Average travel time (s) \\
\hline $0 \%$ & 197.2 \\
\hline $20 \%$ & 200.8 \\
\hline $40 \%$ & 193.1 \\
\hline $60 \%$ & 174.0 \\
\hline $80 \%$ & 160.8 \\
\hline $100 \%$ & 151.1 \\
\hline
\end{tabular}

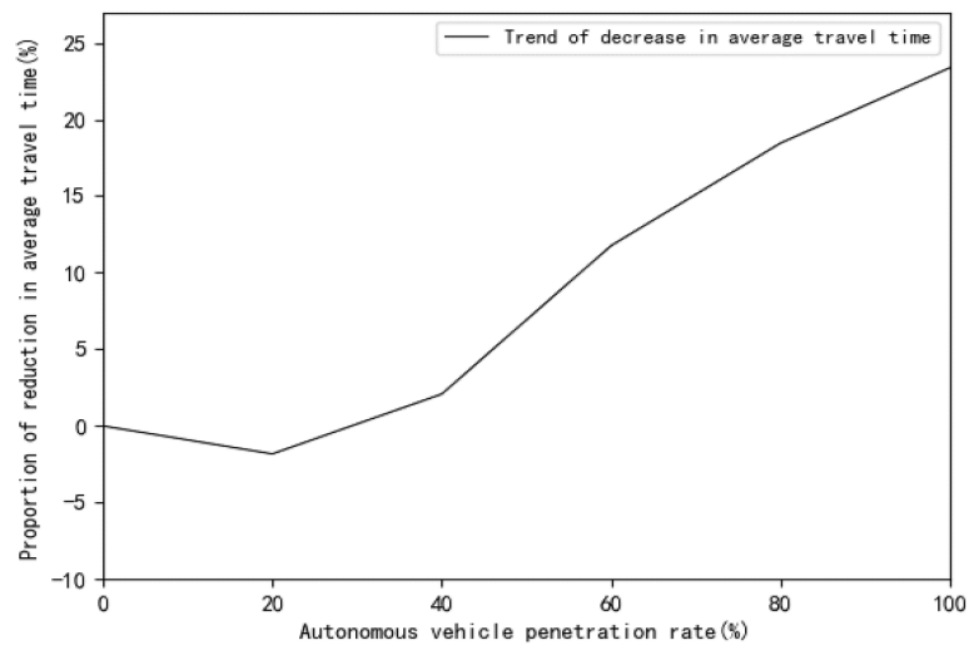

Figure 8: Increased value of average travel time under different penetration scenarios.

As shown in Table IV and Fig. 8, along with the rising of the proportion of autonomous, the average travel time of vehicles in the mixed flow increases firstly and then decreases, the critical penetration rate is $20 \%$. When the proportion of autonomous is $100 \%$, the average travel time is the least, reduced by $46.1 \mathrm{~s}$, or $23.38 \%$, compared with the all ordinary vehicle scenario. It indicates that infiltration of multi-level autonomous can reduce the average travel time and improve the overall operation efficiency of road, but the improvement also strongly associated with autonomous driving proportion.

\section{CONCLUSION}

Based on SUMO and Python co-simulation platform, this paper constructs the driving behaviour rules of L0, L2 and L4 vehicles from a microscopic perspective, and analyses the capacity of mixed traffic flow by quantifying penetration rates of autonomous vehicles. The research results prove that automation technology can effectively improve road traffic conditions. However, in this paper, there is a deviation between the construction of the ordinarymulti-level autonomous traffic flow simulation model and the real, it is assumed that the driving simulation environment is not affected by various factors in real life. In the future, the model parameters can be set more accurately built on the experimental material, and a more reasonable and real traffic environment can be studied.

\section{ACKNOWLEDGEMENTS}

This work was supported by fund projects: Postgraduate Research \& Practice Innovation Program of Jiangsu Province (SJCX20_1117, SJCX21_1420, KYCX21_2999), Construction System Project of Jiangsu Province (2020ZD14, 2018ZD258), Suzhou Social Science Fund (Y2020LX017, Y2020LX025), General Project of Jiangsu Natural Fund (BK20151201, BK20160357) and Philosophy and Social Science Projects of Universities in Jiangsu Province (2018SJA1348). 


\section{REFERENCES}

[1] Sun, J.; Huang, R. H.; Li, L.; Liu, Q. Y.; Li, Y. D. (2020). Integrated simulation test platform for environment perception and planning decision of intelligent vehicle, Journal of System Simulation, Vol. 32, No. 2, 236-246, doi:10.16182/j.issn1004731x.joss.18-0102

[2] Yao, Z.-H.; Jiang, Y.-S. (2018). Integrated connected vehicle simulation platform of Vissim and Python, Computer Simulation, Vol. 35, No. 12, 143-146, doi:10.3969/j.issn.10069348.2018.12.036

[3] Ma, J.; Zhang, L.-Y.; Li, K.-P. (2016). Traffic flow modeling and simulation of urban expressway and its ramp, Computer Simulation, Vol. 33, No. 12, 157-162, doi:10.3969/j.issn.10069348.2016.12.033

[4] Zhang, L.; Ma, J.; Ran, B.; Yan, L. (2017). Traffic multiresolution modeling and consistency analysis of urban expressway based on asynchronous integration strategy, Modelling and Simulation in Engineering, Vol. 2017, Paper 3694791, 19 pages, doi:10.1155/2017/3694791

[5] Hu, M. W.; Zhang, Z. M.; Chen, X. S. (2021). Research on benefits of mixed traffic flow of intelligent connected vehicles, Journal of System Simulation, Vol. 33, No. 9, 2270-2278, doi:10.16182/j.issn1004731x.joss.20-0487

[6] Lu, Q.; Tettamanti, T.; Varga, I. (2018). Impacts of autonomous vehicles on the urban fundamental diagram, Proceedings of the $5^{\text {th }}$ International Conference on Road and Rail Infrastructure, 12651271

[7] Ye, L.; Yamamoto, T.; Morikawa, T. (2018). Heterogeneous traffic flow dynamics under various penetration rates of connected and autonomous vehicle, $21^{\text {st }}$ International Conference on Intelligent Transportation Systems, 555-559, doi:10.1109/ITSC.2018.8569975

[8] Kerschbaumer, A.; Rudigier, M.; Haberl, M.; Hintermayer, B. (2017). Automated driving functions for traffic flow models to assess the traffic situation, Isermann, R. (Ed.), Fahrerassistenzsysteme 2017, Springer Vieweg, Wiesbaden, 1-15, doi:10.1007/978-3-658-19059-0_1

[9] Yao, Z.; Hu, R.; Wang, Y.; Jiang, Y.; Ran, B.; Chen, Y. (2019). Stability analysis and the fundamental diagram for mixed connected automated and human-driven vehicles, Physica A: Statistical Mechanics and its Applications, Vol. 533, Paper 121931, 16 pages, doi:10.1016/j.physa.2019.121931

[10] Arvin, R.; Khattak, A. J.; Kamrani, M.; Rio-Torres, J. (2021). Safety evaluation of connected and automated vehicles in mixed traffic with conventional vehicles at intersections, Journal of Intelligent Transportation Systems, Vol. 25, No. 2, 170-187, doi:10.1080/15472450.2020.1834392

[11] Liu, X. G. (2019). Study on the Application of Tort Liability Caused by Level 3 Self-driving Cars in Road Traffic Accidents, Master Thesis, Suzhou University, Suzhou, doi:10.27351/ d.cnki.gszhu.2019.003284

[12] Hong, W. Q.; Tan, H.; Zhang, H. T. (2021). Development trend of autonomous driving at home and abroad, C-Enterprise Management, Vol. 2021, No. 3, 54-57

[13] Sagir, F.; Ukkusuri, S. V. (2018). Mobility impacts of autonomous vehicle systems, $21^{s t}$ International Conference on Intelligent Transportation Systems, 485-490, doi:10.1109/ ITSC.2018.8569933

[14] Guériau, M.; Dusparic, I. (2020). Quantifying the impact of connected and autonomous vehicles on traffic efficiency and safety in mixed traffic, $23^{\text {rd }}$ International Conference on Intelligent Transportation Systems, 8 pages, doi:10.1109/ITSC45102.2020.9294174

[15] Tursun, M.; Geni, M. (2018). SUMO simulation of oversaturated weaving section and lanechanging model optimization, Journal of Xinjiang University (Natural Science Edition), Vol. 35, No. 1, 96-101, doi:10.13568/j.cnki.651094.2018.01.015

[16] Treiber, M.; Hennecke, A.; Helbing, D. (2000). Congested traffic states in empirical observations and microscopic simulations, Physical Review E, Vol. 62, No. 2, 1805-1824, doi:10.1103/ PhysRevE.62.1805

[17] Qin, Y. Y.; Wang, H.; Wang, W.; Wan, Q. (2017). Driving comfort of traffic flow mixed with cooperative adaptive cruise control vehicles and adaptive cruise control vehicles, Journal of Harbin Institute of Technology, Vol. 49, No. 9, 103-108, doi:10.11918/j.issn.0367-6234.201610006 
[18] Chen, T. (2020). Study on the Traffic Characteristics of Continuous Flow Under the Autonomous Driving Environment, Master Thesis, Southeast University, Nanjing, doi:10.27014/ d.cnki.gdnau.2020.001513

[19] Wang, B. Y.; Li, J. W. (2015). Statistical analysis and countermeasures of China's traffic accidents, Contemporary Economy, Vol. 2015, No. 20, 116-119

[20] Lu, W. (2018). A Study on the Two-lane Traffic Flow Characteristics with the Influence of Driver Behavior, Master Thesis, Lanzhou Jiaotong University, Lanzhou

[21] Milanés, V.; Shladover, S. E. (2014). Modeling cooperative and autonomous adaptive cruise control dynamic responses using experimental data, Transportation Research Part C: Emerging Technologies, Vol. 48, 285-300, doi:10.1016/j.trc.2014.09.001

[22] Milanes, V.; Shladover, S. E. (2016). Handling cut-in vehicles in strings of cooperative adaptive cruise control vehicles, Journal of Intelligent Transportation Systems, Vol. 20, No. 2, 178-191, doi:10.1080/15472450.2015.1016023

[23] Xiao, L.; Wang, M.; van Arem, B. (2017). Realistic car-following models for microscopic simulation of adaptive and cooperative adaptive cruise control vehicles, Transportation Research Record: Journal of the Transportation Research Board, Vol. 2623, No. 1, 1-9, doi:10.3141/2623$\underline{01}$

[24] Yanakiev, D.; Kanellakopoulos, I. (1998). Nonlinear spacing policies for automated heavy-duty vehicles, IEEE Transactions on Vehicular Technology, Vol. 47, No. 4, 1365-1377, doi:10.1109/ 25.728529

[25] Kudarauskas, N. (2007). Analysis of emergency braking of a vehicle, Transport, Vol. 22, No. 3, 154-159

[26] Erdmann, J. (2015). SUMO's lane-changing model, Behrisch, M.; Weber, M. (Eds.), Modeling Mobility with Open Data, Springer, Cham, 105-123, doi:10.1007/978-3-319-15024-6_7

[27] Lackey, N. A. (2019). Simulating Autonomous Vehicles in a Microscopic Traffic Simulator to Investigate the Effects of Autonomous Vehicles on Roadway Mobility, Master Thesis, The Ohio State University, Columbus

[28] Chen, D. S.; Yu, X. X.; Hu, K. Q.; Sun, X.; Xia, Y. Y. (2014). Safety-oriented speed guidance of urban expressway under model predictive control, International Journal of Simulation Modelling, Vol. 13, No. 2, 219-229, doi:10.2507/IJSIMM13(2)CO8

[29] Lu, Q.; Tettamanti, T. (2021). Impacts of connected and automated vehicles on freeway with increased speed limit, International Journal of Simulation Modelling, Vol. 20, No. 3, 453-464, doi:10.2507/IJSIMM20-3-556 